\title{
Electroresistance of $\mathrm{La}-\mathrm{Ca}-\mathrm{MnO}$ Thin Films
}

\author{
P. Cimmperman ${ }^{a}$, V. Stankevič ${ }^{a}$, N. ŽurauskienE ${ }^{a, *}$, \\ S. Balevičius ${ }^{a}$, F. Anisimovas ${ }^{a}$, J. PARŠeliūnas ${ }^{a}$, \\ O. KiprijanoviČ ${ }^{a}$ AND L.L. Altgilbers ${ }^{b}$
}

${ }^{a}$ Semiconductor Physics Institute, A. Goštauto 11, Vilnius, Lithuania ${ }^{b}$ US Army Space and Missile Defense Command, Huntsville AL, USA

Epitaxial, textured, and polycrystalline $\mathrm{La}_{0.7} \mathrm{Ca}_{0.3} \mathrm{MnO}_{3}$ films, having about $150 \mathrm{~nm}$ thickness, were prepared by pulsed laser deposition techniques onto (110) $\mathrm{NdGaO}_{3}, \mathrm{MgO}$ and lucalox substrates and investigated using $10 \mathrm{~ns}$ duration, $0.5 \mathrm{~ns}$ rise time electrical pulses having amplitude up to $500 \mathrm{~V}$. Electroresistance of the films $[R(E)-R(0)] / R(0)$ was investigated up to $80 \mathrm{kV} / \mathrm{cm}$ electric field strengths in temperatures ranging from $300 \mathrm{~K}$ to $4.2 \mathrm{~K}$. Strong (up to $93 \%$ ) negative electroresistance was obtained in polycrystalline $\mathrm{La}_{0.7} \mathrm{Ca}_{0.3} \mathrm{MnO}_{3}$ films prepared on $\mathrm{MgO}$ and lucalox substrates. The epitaxial films grown on $\mathrm{NdGaO}_{3}$ substrate demonstrated only a small resistance change due to Joule heating induced by a current pulse. It was concluded that electroresistance manifests itself in strongly inhomogeneous manganites films exhibiting a large number of structural imperfections producing ferromagnetic tunnel junction nets.

PACS numbers: 73.50.Gr, 73.50.Lw, 73.63.Bd

\section{Introduction}

It has been observed that manganites exhibiting colossal magnetoresistance (CMR) phenomenon are also sensitive to electric fields that induce strong decreases in the electrical resistance of these materials. This effect was named electroresistance (ER) and was studied in $\mathrm{Pr}_{0.7} \mathrm{Ca}_{0.3} \mathrm{MnO}_{3}$ [1-3], $\mathrm{Nd}_{0.7} \mathrm{Sr}_{0.3} \mathrm{MnO}_{3}$ [4], and $\mathrm{La}_{1-x} \mathrm{Ca}_{x} \mathrm{MnO}_{3}$ system. The ER was detected in bulk polycrystalline $\mathrm{La}_{0.7} \mathrm{Ca}_{0.3} \mathrm{MnO}_{3}$ [5], single crystals of $\mathrm{La}_{0.82} \mathrm{Ca}_{0.18} \mathrm{MnO}_{3}$ [6], and epitaxial

* corresponding author; e-mail: zurausk@pfi.lt 
$\mathrm{La}_{0.7} \mathrm{Ca}_{0.3} \mathrm{MnO}_{3}$ films [7]. The effect of short pulsed electric fields on the resistance of thin $\mathrm{La}_{0.67} \mathrm{Ca}_{0.33} \mathrm{MnO}_{3}$ films was investigated in [8,9]. As a result, a fast fault current limiter with subnanosecond time turn on time was suggested $[10,11]$. However, the reasons for the origin of ER are still unclear, thus making difficult the further development of this device and wider application of the ER phenomenon for other field effect devices. Moreover, it seems that the nature of ER is different in bulk ceramics, single crystals, and thin films of manganites. According to [5], the strong decrease in resistance that occurs with the increase of electric field is due to the Frenkel-Poole effect in the interfacial regions of the manganite ceramic. The ER in single crystal samples was explained as electric field induced CMR [6] which takes place in narrow channels of the crystal because of current percolation in medium consisting of two phases with different electronic densities. The explanation of ER in epitaxial films was also based on a two-phase system containing high conductivity ferromagnetic regions (FM) and low conductivity charge ordering (CO) regions [7]. However, in this case, it was suggested that the electric field modifies the volume fractions of the FM and CO components. It has to be noted that the effect of experimental conditions, i.e. dc or pulsed current at which ER appears, was not taken into consideration.

In this paper, we report on an experimental study of short electrical pulse induced $\mathrm{ER}$ in $\mathrm{La}_{0.67} \mathrm{Ca}_{0.33} \mathrm{MnO}_{3}$ films containing different imperfection densities and demonstrating that the very large (up to $93 \%$ ) ER effect is caused by effects of strong electric fields on the resistance of these imperfections.

\section{Sample preparation}

The films were grown onto (110) $\mathrm{NdGaO}_{3}$ (NGO), $\mathrm{MgO}$ (MGO), and lucalox substrates by on-axis pulsed laser deposition using $\mathrm{La}_{0.67} \mathrm{Ca}_{0.33} \mathrm{MnO}_{3}$ target synthesized by a solid state reaction technique. $\mathrm{A} \mathrm{Nd}^{3+}$ :YAG laser, operated in a doubled frequency mode $(\lambda=532 \mathrm{~nm})$, was used for this deposition. Laser pulse energy was chosen to be $50 \mathrm{~mJ}$. During the deposition, the temperature of the substrate was kept at $750^{\circ} \mathrm{C}$. The growth was followed by annealing in $1 \mathrm{~atm}$. pressure oxygen at $750^{\circ} \mathrm{C}$ for $30 \mathrm{~min}$. After this, the film was cooled down to room temperature for 3 hours. The thickness of the $\mathrm{La}_{0.67} \mathrm{Ca}_{0.33} \mathrm{MnO}_{3}$ films was about $140 \mathrm{~nm} . \mathrm{NdGaO}_{3}$ and $\mathrm{MgO}$ substrates were single crystals with orthorhombic and cubic unit cell structures, respectively. The difference between the plane lattice constants of the $\mathrm{NdGaO}_{3}$ and $\mathrm{MgO}$ substrates and the bulk $\mathrm{La}_{0.67} \mathrm{Ca}_{0.33} \mathrm{MnO}_{3}$ were $1 \%$ and $9 \%$, respectively. Lucalox substrate was a polycrystalline structure.

The samples, having a length of $5 \mathrm{~mm}$, were designed in a co-planar shape with the width ranging from $1.5 \mathrm{~mm}$ to $3 \mathrm{~mm}$. Four $\mathrm{Ag}$ tapes having $1 \mathrm{~mm}$ width and spaced by $50 \mu \mathrm{m}$ gap were deposited onto the film by thermal evaporation at $200^{\circ} \mathrm{C}$. After this, the films were annealed for 1 hour at $1 \mathrm{~atm}$. pressure in argon gas at $400^{\circ} \mathrm{C}$. The resistance of the samples for low value dc currents was measured 
by a standard four-probe method. In order to investigate the ER of the samples exposed to strong pulsed electric fields, the samples were connected in series to a $50 \Omega$ impedance high frequency $(18 \mathrm{GHz})$ transmission line and pulsed by $5-10 \mathrm{~ns}$ duration, $0.5 \mathrm{~ns}$ rise time rectangular shape electrical pulses [9]. The amplitude of these pulses was changed from $10 \mathrm{~V}$ to $500 \mathrm{~V}$. Measurements of dc resistivity and ER were performed at temperatures ranging from $4.2 \mathrm{~K}$ to $300 \mathrm{~K}$, using a closed cycle helium gas cryocooler.

\section{Results}

\subsection{DC resistivity measurements}

Figure 1 shows a typical electrical resistivity $(\rho)$ vs. temperature $(T)$ dependence for $\mathrm{La}_{0.67} \mathrm{Ca}_{0.33} \mathrm{MnO}_{3}$ films prepared on different substrates. Films deposited on NGO substrate was investigated using a $0.86 \mathrm{~mA}$ excitation current. In the case of films deposited on MGO and lucalox substrates, this current was $0.1 \mathrm{~mA}$. It is evident that $\rho=f(T)$ for all the investigated films exhibits a maximum at a certain critical temperatures $T_{\mathrm{m}}$. For films grown on NGO, MGO, and lucalox

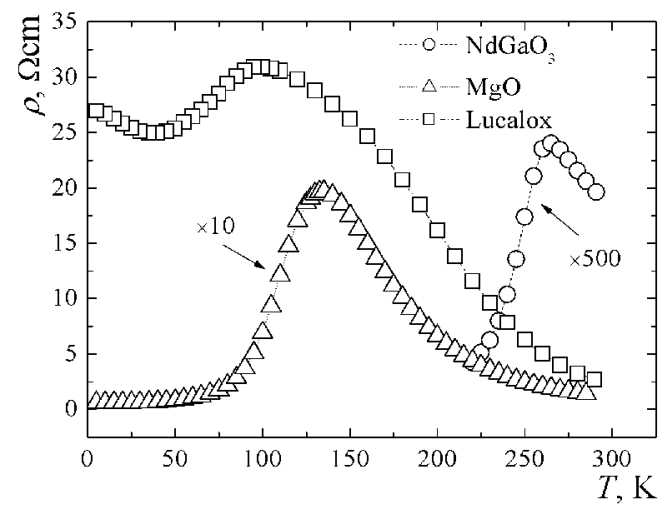

Fig. 1. Resistivity vs. temperature dependence for $\mathrm{La}_{0.67} \mathrm{Ca}_{0.33} \mathrm{MnO}_{3}$ films prepared on different substrates.

substrates, this temperature is $265 \mathrm{~K}, 135 \mathrm{~K}$, and $100 \mathrm{~K}$, respectively. At temperatures below $T_{\mathrm{m}}$, the resistivity of the films deposited on NGO and MGO substrates decreases with temperature, while for films prepared on lucalox substrate, the $\rho=f(T)$ dependence has a minimum at $T=35 \mathrm{~K}$. At this temperature, the metallic-like resistivity behavior changes to a semiconductor state. Figure 1 also demonstrates that an increase in $T_{\mathrm{m}}$ is always accompanied with a significant decrease in the resistivity of the film. The largest resistivity $\left(\rho_{\max }=30.9 \Omega \mathrm{cm}\right)$ corresponds to the lowest $T_{\mathrm{m}}=100 \mathrm{~K}$ obtained for films prepared on lucalox substrate. In the case of $\mathrm{MGO}$ and $\mathrm{NGO}$, the $\rho_{\max }$ was $1.97 \Omega \mathrm{cm}$ and $50 \mathrm{~m} \Omega \mathrm{cm}$, respectively. 


\subsection{Electroresistance induced by pulsed electric field}

The ER using short electric pulses was investigated at temperatures ranging from $4.2 \mathrm{~K}$ to $300 \mathrm{~K}$. We defined the value of the electroresistance $(E R)$ as follows:

$$
E R=\frac{R(E)-R(0)}{R(0)} \times 100 \%,
$$

where $R(E)$ is the resistance of the sample effected by pulsed strong electric fields and $R(0)$ is the resistance of the sample effected by pulsed electric fields, the value of which does not exceed $0.5 \mathrm{kV} / \mathrm{cm}$. It was found that $R(0)$ is the same as that measured using dc current.

Figure 2 depicts the $E R$ effect in $\mathrm{La}_{0.67} \mathrm{Ca}_{0.33} \mathrm{MnO}_{3}$ films deposited on $\mathrm{NGO}$ substrate. The resistance change was measured $2 \mathrm{~ns}$ after the start of the pulse. As can be seen below, if the temperature is approximately equal to $260 \mathrm{~K}$, the $E R$ is positive if electric field strength does not exceed $25 \mathrm{kV} / \mathrm{cm}$, however at higher temperatures it is very small (less than 10\%) and negative. The positive $E R$ vs. $E$ dependence increase is higher at low temperatures and becomes less abrupt at temperatures close to $T_{\mathrm{m}}$. At these temperatures, this dependence exhibits a maximum at electric field strengths of $20 \mathrm{kV} / \mathrm{cm}$.

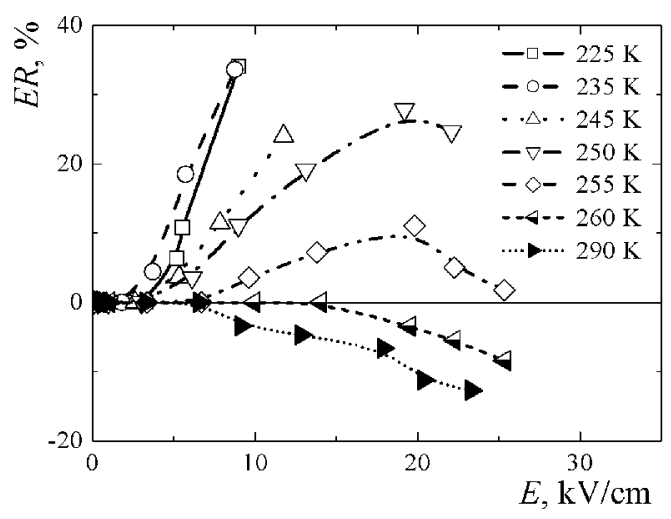

Fig. 2. Electroresistance vs. electric field strength dependence for $\mathrm{La}_{0.67} \mathrm{Ca}_{0.33} \mathrm{MnO}_{3}$ films deposited on $\mathrm{NdGaO}_{3}$ substrate.

The $E R$ vs. electric field strength behavior of films grown on MGO substrates strongly differs from that obtained for films on NGO substrate. The highest negative $E R$ was obtained at temperatures around $T_{\mathrm{m}}$. Decrease or increase in temperature from $T_{\mathrm{m}}$ has a smaller effect on the $E R$. A typical $E R$ vs. $E$ dependence of negative $E R$ effects slightly below $T_{\mathrm{m}}$ demonstrates the decrease in $E R$ with an increase in $E$. This increase at high electric field strength tends to saturate. The saturation becomes more noticeable at lower temperatures and at critical fields at which the saturation starts to decrease as the temperature decreases. Starting from temperatures at around $80 \mathrm{~K}$, the $E R$ vs. $E$ dependence 
exhibits a minimum. In this case, the $E R$ value increases only up to a certain critical electric field; for higher fields the $E R$ value decreases with an increase in $E$. This increase becomes so strong at $60 \mathrm{~K}$ that the $E R$ changes its sign from negative to positive (see Fig. 3a). The increase in temperature above $T_{\mathrm{m}}$ also makes the $E R$ vs. $E$ dependence less abrupt. However, in this case, the saturation of the $E R$ vs. $E$ dependence at higher electric fields disappears. At high temperatures, this dependence is very similar to that obtained in films prepared on NGO substrates (see Fig. 3b).
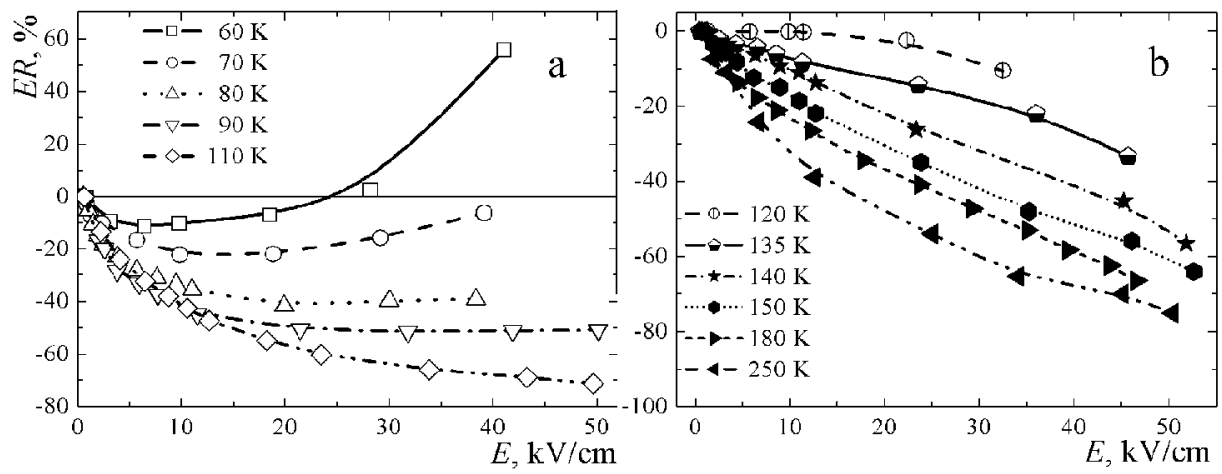

Fig. 3. (a, b) Electroresistance vs. electric field strength dependence for $\mathrm{La}_{0.67} \mathrm{Ca}_{0.33} \mathrm{MnO}_{3}$ films deposited on $\mathrm{MgO}$ substrate.

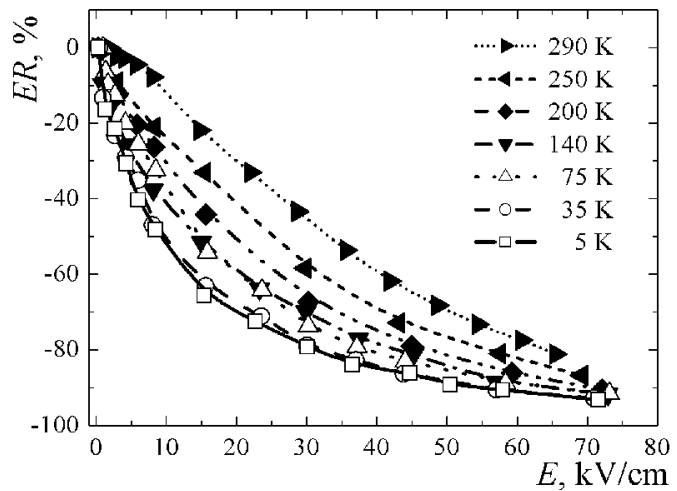

Fig. 4. Electroresis tance vs. electric field strength dependence for $\mathrm{La}_{0.67} \mathrm{Ca}_{0.33} \mathrm{MnO}_{3}$ films deposited on lucalox substrate.

The results of ER effect investigation in films grown on lucalox substrate is presented in Fig. 4. It is evident that only negative electroresistance appears in this case over the entire investigated temperature range. The value of $E R$ increases with the increase in electric field strength and with the decrease in film temperature. The highest value of $E R(93 \%)$ was obtained at $5 \mathrm{~K}$. In this case, the shape of the $E R$ vs. $E$ curve is similar to that obtained in films prepared on 
MGO substrate and kept at temperatures around $T_{\mathrm{m}}$. The changes in the shape of the $E R$ vs. $E$ curves with the increase in temperature are also similar to films deposited on MGO substrate, if the temperature increases above $T_{\mathrm{m}}$.

\section{Discussion}

Before discussing possible reasons for the origin of electroresistance in the investigated films, it is necessary to discuss the results of the resistance vs. temperature dependence investigated for the dc current regime. It was found that films prepared on NGO substrate had low resistance and a high critical temperature $T_{\mathrm{m}}=265 \mathrm{~K}$. Such behavior is typical of epitaxial film grown on a substrate with a small mismatch of lattice parameters between substrate and film (a lattice parameter difference between bulk LCMO and NGO is only 1\%). In the case of the MGO substrate, this mismatch is significant $(9 \%)$ and a large number of dislocations producing grain boundaries (GBs) necessary to absorb the misfits are formed [12]. The film grown on polycrystalline lucalox substrate has to contain more imperfections than that prepared on MGO substrate, because in addition to the lattice mismatch, the lucalox substrate is of granular structure. Thus, if the lattice mismatch generates GB in the same direction as the orientated crystallites, like in the MGO case, the lucalox substrate creates additional GBs between the disorientated crystallites [13]. A higher resistivity and strong differences between the $R(T)$ dependence for the films prepared on lucalox and MGO substrates confirm this assumption.

Small values of $E R$ and $E R$ vs. $E$ dependence in films grown on NGO substrate can be explained by current induced heating of the film due to a strong electric field pulse action. Positive and negative $E R$ obtained below and above $T_{\mathrm{m}}$, respectively, show that Joule heating could be the main reason of this effect. Let us suppose that $R(T)$ is the same for all electric field strength values, heating is adiabatic and the film resistance dependence on temperature is linear. It is almost true for relatively narrow temperature ranges and short electrical pulse durations. In this case, the temperature increase of the film during the electrical pulse action can be obtained from the following formula:

$$
\Delta T=\frac{U^{2} t}{R_{\mathrm{a}} c m}
$$

where $U$ is the voltage, $t$ is the time of current flow, and $c$ and $m$ are the heat capacity and mass of the film, respectively. $R_{\mathrm{a}}$ is the average resistance of the sample. Figure 5 (triangular dots) shows the temperature increase calculated when $t=2 \mathrm{~ns}$ and when the electric field strength was $20 \mathrm{kV} / \mathrm{cm}$. The circular dots are experimental data points (see Fig. 1) and demonstrate how much the temperature has to increase in order to get a corresponding electric field induced resistance change (see Fig. 2). As can be seen, experimental data and calculations are in relatively good agreement at temperatures below $T_{\mathrm{m}}$. However, the difference at $T>T_{\mathrm{m}}$ demonstrates that in addition to Joule heating, there is a non-thermal 


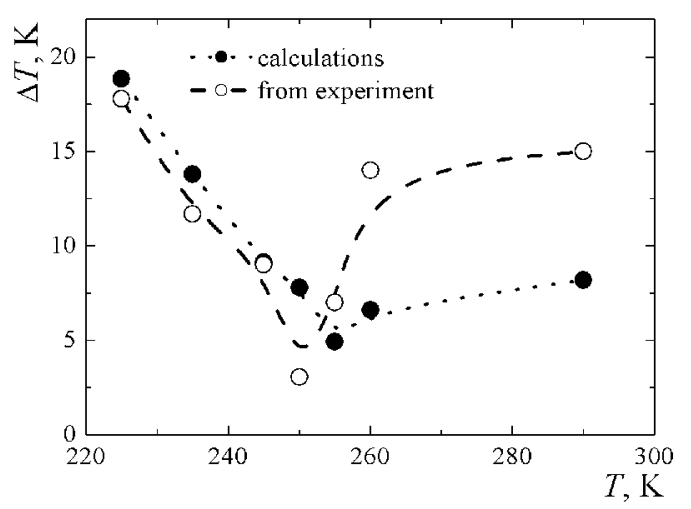

Fig. 5. Calculated (solid dots) electric pulse induced temperature increase of $\mathrm{La}_{0.67} \mathrm{Ca}_{0.33} \mathrm{MnO}_{3}$ film deposited on $\mathrm{NdGaO}_{3}$ substrate (applied electric field $20 \mathrm{kV} / \mathrm{cm}$ ). Circular dots show temperature increase obtained from the resistance vs. temperature dependence.

origin of the strong electric field effect, such as field assisted hopping [14], which manifests itself when the film is transformed to semiconductor-like behavior.

As it is shown in Fig. 1, films prepared on MGO substrates have a significantly lower $T_{\mathrm{m}}=135 \mathrm{~K}$ and 50 times higher resistivity than films deposited on NGO. In this case, the main reason for the electroresistance phenomenon (negative and positive) at $T \gg T_{\mathrm{m}}$ and $T \ll T_{\mathrm{m}}$ is also Joule heating, while a large negative electroresistance nearby $T_{\mathrm{m}}$ is of pure electronic origin and probably is determined by charge carriers tunneling across high resistivity grain boundaries [13]. Most probably, the same or similar effect is responsible for ER phenomenon in high resistivity films fabricated using polycrystalline lucalox substrate. In this case grain boundaries formed between disoriented manganite crystals could play a significant role in determining the film's electrical properties. The main voltage drop in this case is concentrated at these imperfections, where the resistivity is less sensitive to magnetic ordering. For this reason, only a strong negative electroresistance phenomenon was observed for the investigated temperature range from $5 \mathrm{~K}$ to $300 \mathrm{~K}$.

\section{Summary}

The electroresistance effect caused by nanosecond duration electrical pulses in high quality $\mathrm{La}_{0.67} \mathrm{Ca}_{0.33} \mathrm{MnO}_{3}$ films grown onto (110) NGO substrate and having a small number of imperfections for the investigated temperature range is mostly due to Joule heating. However, the resistance changes close to and above $T_{\mathrm{m}}$ is the result of both Joule heating and non-thermal strong electric field effects. The films prepared on MGO substrates have significantly lower $T_{\mathrm{m}}=135 \mathrm{~K}$ and 50 times higher resistivity than films deposited on NGO. Most probably, this is caused by grain boundaries formed due to the misfit between the film and 
substrate lattice constants. In this case, the main reason for the ER phenomenon (negative and positive) at $T \gg T_{\mathrm{m}}$ and $T \ll T_{\mathrm{m}}$ is also Joule heating, while large negative $E R$ values near $T_{\mathrm{m}}$ is of pure electronic origin and probably is determined by charge carrier tunnelling across high resistivity grain boundaries. The ER phenomenon in films fabricated using polycrystalline lucalox substrate is also associated with strong electric fields on the GB, which, most probably, are formed between disoriented manganite crystals. For this reason, only a strong negative ER phenomenon was observed over the investigated temperature range from $5 \mathrm{~K}$ to $300 \mathrm{~K}$.

\section{Acknowledgments}

The work was supported by the Lithuanian Science and Studies Foundation under the contract No. K-058.

\section{References}

[1] Asaitsu, Y. Tomioka, H. Kuwahara, Y. Tokura, Nature 388/3, 50 (1997).

[2] S.Q. Lui, N. Wu, A. Ignatiev, Appl. Phys. Lett. 76, 2749 (2000).

[3] Sh. Srivastava, N.K. Pandey, P. Padhan, R.C. Budhani, Phys. Rev. B 62, 13868 (2000).

[4] S.B. Ogale, V. Talyansky, C.H. Chen, R. Ramesh, R.L. Greene, T. Venkatesan, Phys. Rev. Lett. 77, 1159 (1996).

[5] N.I. Solin, A.A. Camochvalov, S.V. Naumov, Fiz. Tverd. Tel. 40, 1881 (1998).

[6] V. Markovich, E. Rozenberg, Y. Yuzhelevski, G. Jung, G. Gorodetsky, Appl, Phys. Lett. 78, 3499 (2001).

[7] T. Wu, S.B. Ogale, J.E. Garrison, B. Nagaraj, A. Biswas, Z. Chen, R.L. Greene, R. Ramesh, T. Venkatesan, Phys. Rev. Lett. 86, 5998 (2001).

[8] S. Balevičius, O. Kiprijanovič, A. Jukna, F. Anisimovas, A. Abrutis, V. Plaušinaitienè, J. Paršeliūnas, B. Vengalis, N. Žurauskienè, Mater. Sci. Forum 384-385, 309 (2002).

[9] O. Kiprijanovič, S. Balevičius, A. Jukna, E.E. Tornau, V. Pyragas, B. Vengalis, F. Anisimovas, in: Proc. Inter. Conf. TFDOM, Vilnius (Lithuania), September 2000, Eds. B. Vengalis, A. Abrutis, Vilnius University Press, Vilnius 2000, p. 122.

[10] S. Balevičius, O. Kiprijanovič, F. Anisimovas, B. Vengalis, N. Žurauskienè, L.L. Altgilbers, J. Phys. IV (France) 11, 91 (2001).

[11] L.L. Altgilbers, S. Balevičius, O. Kiprijanovič, V. Pyragas, E.E. Tornau, A. Jukna, B. Vengalis, F. Anisimovas, in: Proc. 13th IEEE Intern. Pulse Power Conf., Las Vegas (Nevada), June 2001, Vol. 2, 2002, p. 1782.

[12] O.I. Lebedev, J. Verbeeck, G. Van Tendeloo, O. Shapoval, A. Belenchuk, V. Moshnyaga, B. Damashcke, K. Samwer, Phys. Rev. B 66, 104421 (2002).

[13] R. Gross, L. Alff, B. Buchner, B.H. Freitag, C. Hofener, J. Klein, Yafeng Lu, W. Made, J.B. Philipp, M.S.R. Rao, P. Reutler, S. Ritter, S. Thienhaus, S. Uhlenbruck, B. Wiedenhorst, J. Magn. Magn. Mater. 211, 150 (2000).

[14] R.M. Hill, Phylos. Mag. 24, 1307 (1971). 